\title{
Percutaneous or Side-Arm Graft Right Subclavian Artery Cannulation via Median Sternotomy
}

\author{
Corrado Cavozza, MD ${ }^{1}$ Antonio Campanella, MD ${ }^{1}$ Pellegrino Pasquale, MD ${ }^{1}$ Andrea Audo, MD ${ }^{1}$ \\ ${ }^{1}$ Department of Cardiothoracic and Vascular Surgery, Cardiac \\ Surgery, Santissimi. Antonio e Biagio e Cesare Arrigo Hospital, \\ Alessandria, Italy

\begin{abstract}
Address for correspondence Corrado Cavozza, MD, Department of Cardiothoracic and Vascular Surgery, Cardiac Surgery, SS. Antonio e Biagio e Cesare Arrigo Hospital, Via Venezia 16, 15121, Alessandria,
\end{abstract} \\ Italy (e-mail: cc220564@gmail.com).
} AORTA 2019;7:150-153.
Abstract
Keywords
- aortic dissection
- cannulation
- right subclavian artery cannulation

Several cannulation sites alternative to the ascending aorta, such as femoral, right axillary, carotid, innominate artery, and, less commonly, apical sites, have been proposed. Cannulation of the right subclavian artery, through sternotomy, is one possible means of establishing cardiopulmonary bypass, hence avoiding a second surgical incision. In our experience, cardiopulmonary bypass flow was adequate and circulatory arrest with antegrade cerebral perfusion was successfully performed in all cases. There was no in-hospital mortality.

\section{Introduction}

When cannulation of the ascending aorta is not advisable, such as in cases of Type-A aortic dissection, alternatives including femoral, right axillary, carotid, and, less commonly, apex of the left ventricle have been proposed. ${ }^{1-4}$

Cannulation of the femoral vessels carries risk of poor perfusion and retrograde thrombotic embolism. ${ }^{5}$ The cardiac apex can be very fragile. Surgical approach to right axillary artery may be time consuming, especially in obese patients, and this should be weighed against the urgency of the operation. In addition, injury to the brachial plexus, arm hyperperfusion or seroma formation, although rare in experienced hands, are potential complications. Carotid artery is often affected by dissection. Use of the innominate artery, provided that it is not dissected, offers advantages similar to those of the right axillary artery, while eliminating some of the concerns over the added length of the procedure. ${ }^{1,4}$ When the innominate artery is not suitable for arterial cannulation, the right subclavian artery, uncommonly affected by the acute dissection process, may be cannulated either directly or by sewing an end-to-side 8 -mm polyester graft to the artery. This can permit systemic flow with antegrade perfusion without creating any other surgical incision than sternotomy.

received

July 20, 2017

accepted after revision

November 2, 2019

\section{Techniques and Results}

Twenty consecutive patients with Type A aortic dissection received cannulation of the right subclavian artery, directly through percutaneous cannula placement or using a dacron graft, after sternotomy, before pericardial incision (- Fig. 1; - Table 1). Blood pressure in both arms and core temperature were invasively monitored. Median sternotomy is extended to the right neck (-Fig. 2), the innominate vein is identified and encircled with an umbilical tape, allowing the vein to be retracted inferiorly. The innominate artery is exposed, and dissected up to the bifurcation and subclavian artery. The subclavian artery is identified and dissected. The first part of the right subclavian artery passes from the bifurcation of the right brachiocephalic trunk deep to the right sternocostal joint to the medial edge of the scalenus anterior muscle and is crossed by the vagus nerve near brachiocephalic artery division. Exposure is facilitated with a retractor like farabeuf. The right vagus descends within the carotid sheath between the internal jugular vein and the internal and common carotid artery, then crosses anterior to the first part of the subclavian artery at the lower margin and gives off its right recurrent laryngeal branch surrounding the artery (-Fig. 3). The nerve receives close attention from surgeons because the nerve is at Tel: $+1(212) 760-0888$

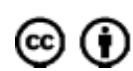

Copyright $\odot 2019$ by Thieme Medical Publishers, Inc., 333 Seventh Avenue, New York, NY 10001, USA.

License terms

10.1055/s-0039-3401019. ISSN 2325-4637. 


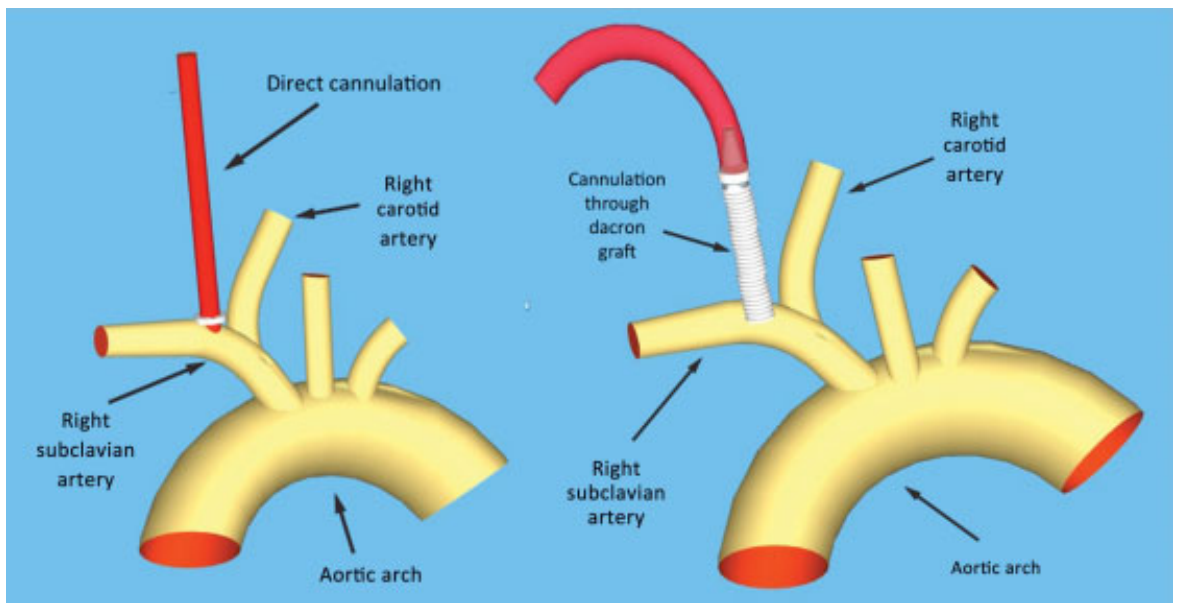

Fig. 1 Schematic representation of percutaneous or through graft subclavian artery cannulation.

Table 1 Patients characteristics

\begin{tabular}{|c|c|}
\hline Variables & Value \\
\hline No. & 20 \\
\hline Mean age in y (range) & $74 \pm 7.5$ \\
\hline Male & 12 \\
\hline Preoperative shock & 5 \\
\hline Preoperative CPA & 1 \\
\hline \multicolumn{2}{|l|}{ Organ malperfusion: } \\
\hline Cerebral & 4 \\
\hline Coronary & 3 \\
\hline Kidney & 7 \\
\hline Spinal cord & 5 \\
\hline Leg & 6 \\
\hline Aortic regurgitation & 18 \\
\hline \multicolumn{2}{|l|}{ Location of primary tear: } \\
\hline Aortic arch & 2 \\
\hline Ascending aorta & 18 \\
\hline Cardiopulmonary bypass time (min) & $183 \pm 82$ \\
\hline Circulatory arrest time (min) & $38.6 \pm 15$ \\
\hline Lowest tympanic temperature $\left({ }^{\circ} \mathrm{C}\right)$ & $24.6 \pm 1.3$ \\
\hline \multicolumn{2}{|l|}{ Concomitant procedures: } \\
\hline Coronary artery bypass grafting & 2 \\
\hline Aortic valve replacement & 5 \\
\hline Root replacement & 3 \\
\hline Conversion to TAR & 4 \\
\hline Reexploration for surgical bleeding & 3 \\
\hline \multicolumn{2}{|l|}{ Major adverse events } \\
\hline Hospital death & 0 \\
\hline Stroke & 2 \\
\hline Paraplegia & 1 \\
\hline Respiratory failure & 2 \\
\hline Permanent hemodialysis & 1 \\
\hline
\end{tabular}

Abbreviations: CPA, perioperative cardiac arrest; $\mathrm{CPB}$, cardiopulmonary bypass; TAR, total arch replacement.

Note: respiratory failure means postoperative pneumonia, pneumothorax, and tracheotomy.

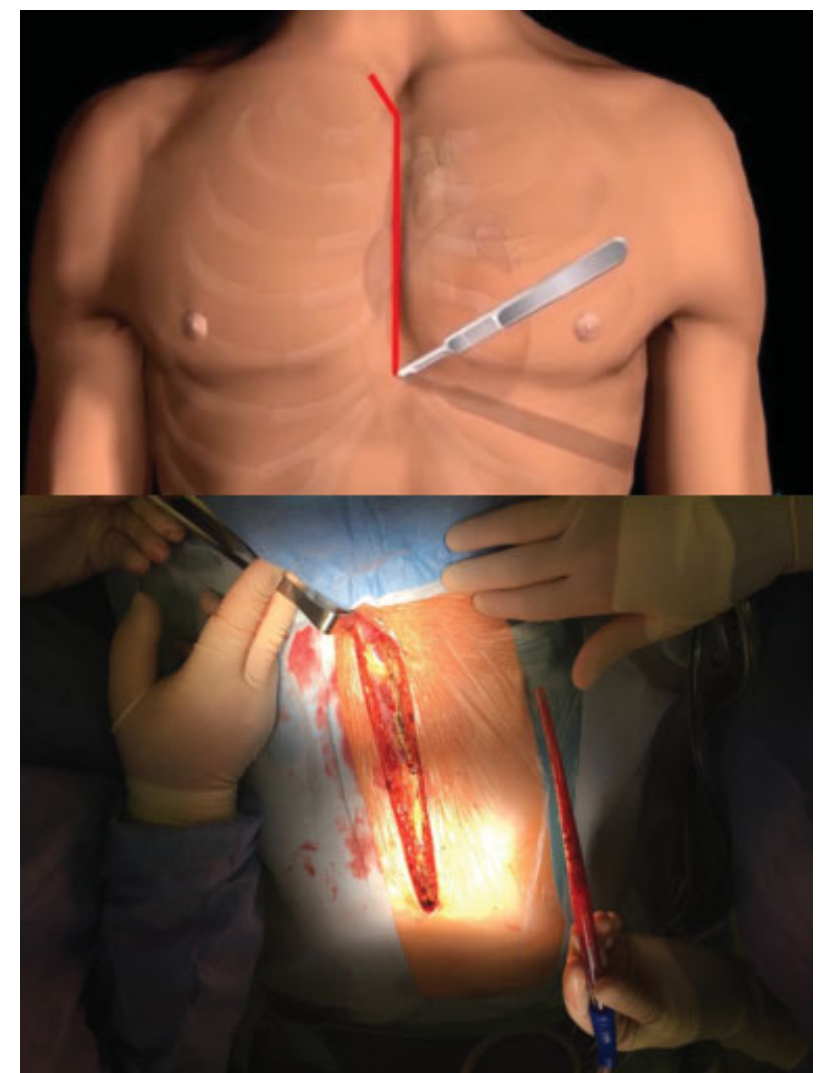

Fig. 2 Sternotomy incision with neck extension.

risk for injury that may prevent with appropriate surgical exposure. After heparinization, the subclavian artery can be cannulated directly percutaneous if transverse diameter is suitable distally to the innominate artery bifurcation. A 20 or 18 French OptiSiteTM arterial cannula (Edwards Lifesciences, Irvine, CA) via Seldinger technique or through a skin incision a 22 French DLP Flexible Arch Arterial Cannula (Medtronic, Inc., Minneapolis, MN) is inserted. Afterwards longitudinal arteriotomy is performed and an 8-mm dacron graft anastomosed, after partially vascular clamp occlusion, taken care not to damage vagus nerve that was easily moved away (-Fig. 4). 


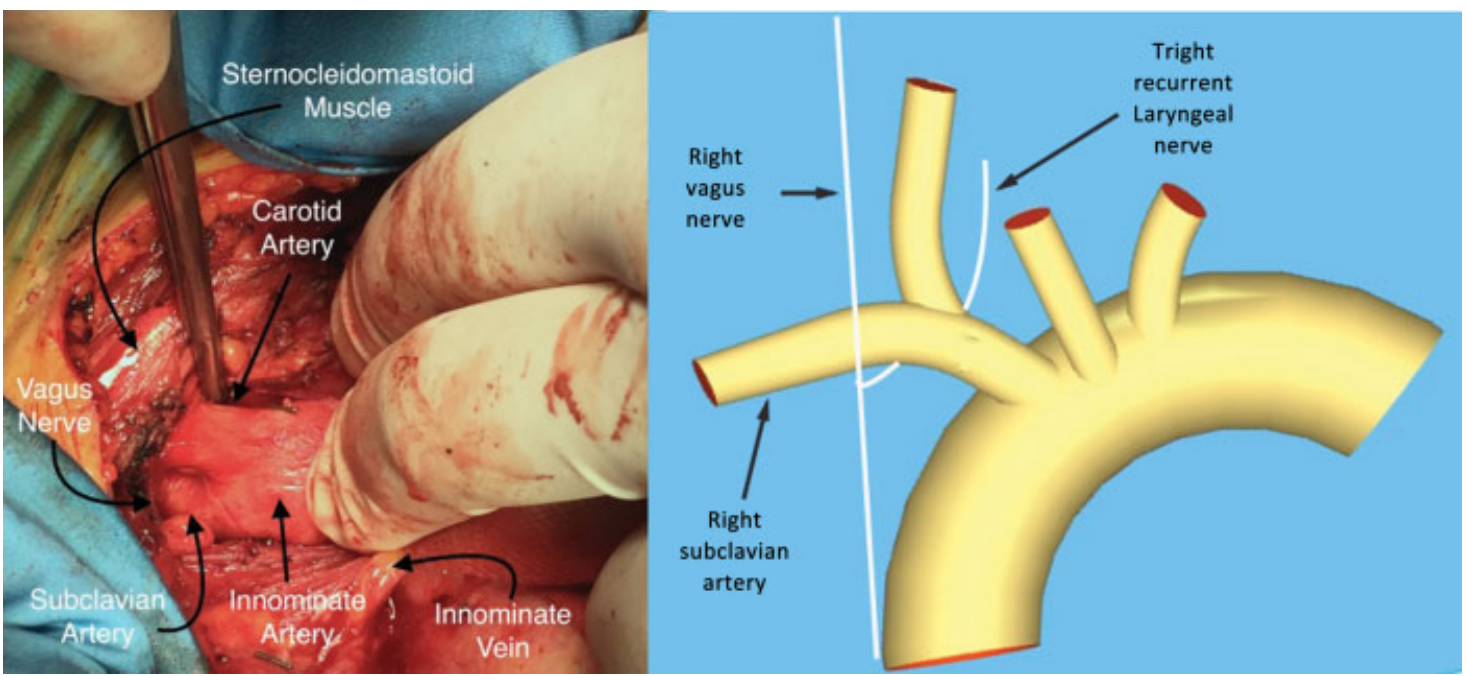

Fig. 3 The vagus nerve then crosses anterior to the first part of the subclavian artery at the lower margin and gives off its right recurrent laryngeal branch surrounding the artery.

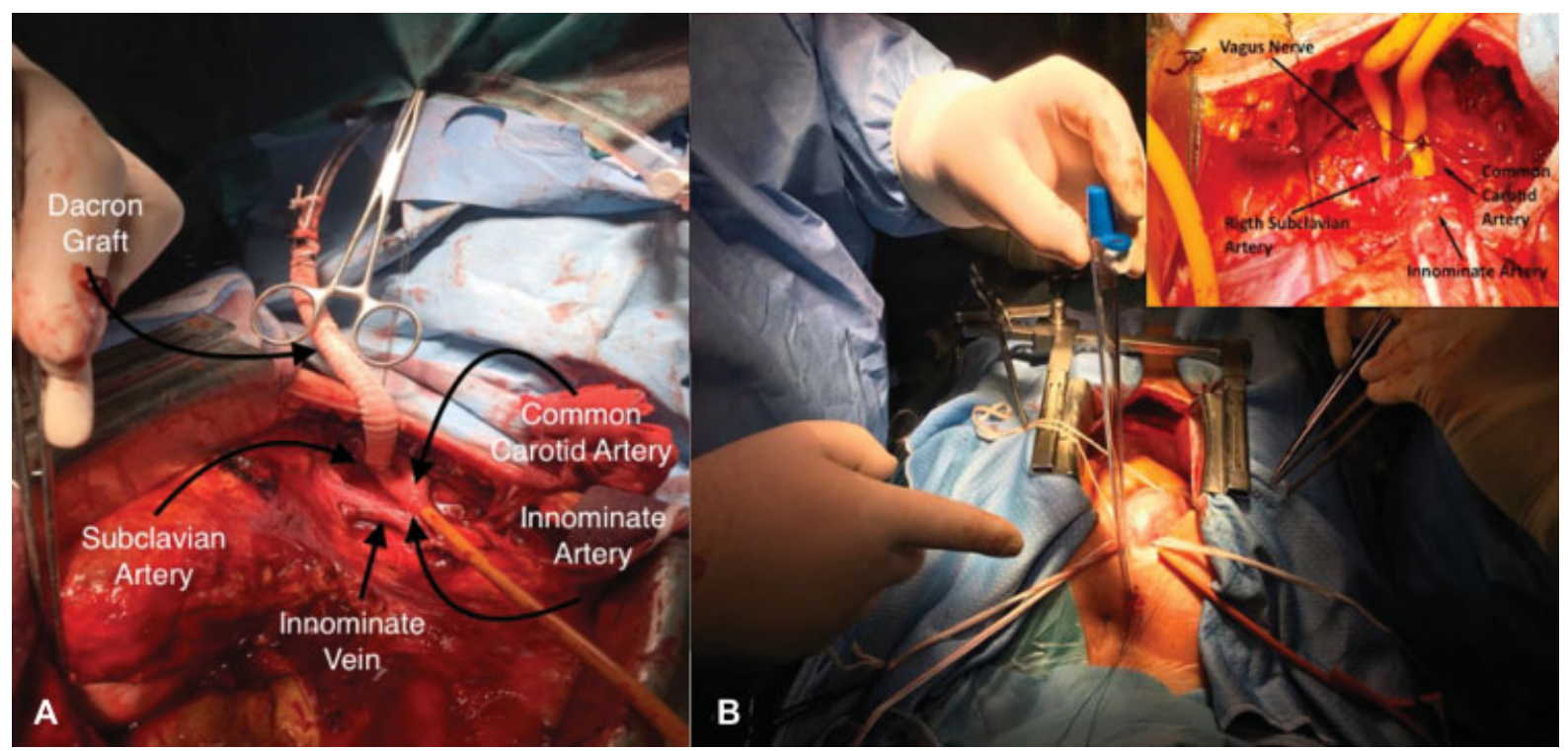

Fig. 4 (A) Rigth subclavian artery cannulation trough side graft. (B) Percutaneous right subclavian artery cannulation.

The arterial line can be and venous cannulation was performed as appropriate. An adequate pump flow rate was achieved. No instances of high pressure in the arterial line were observed. Core temperature was decreased to $26^{\circ} \mathrm{C}$ and after stopping systemic perfusion, unilateral antegrade cerebral perfusion was performed at $10 \mathrm{~mL} / \mathrm{kg}$, via the subclavian artery. To assess brain perfusion, we used solvent infrared spectroscopy capillary saturation by a dual sensor for NIRS (near infrared spectroscopy). Eight patients underwent bilateral perfusion because of unilateral drop in saturation to less than $20 \%$ of the baseline value of NIRS. Right radial artery blood pressure was maintained between 50 and $60 \mathrm{~mm} \mathrm{Hg}$. Mean cardiopulmonary bypass time was $183 \pm 82$ minutes. Mean circulatory arrest time was $38.6 \pm 15$ minutes. There were no cases of traumatic dissection of the subclavian artery. There were no right arm vascular complications and in-hospital mortality. Three patients showed temporary neurological dysfunction defined as presence of reversible postoperative motor deficit, confusion, agitation, or transient delirium. The brain computed tomography scan was normal and all symptoms were resolved before discharge. One patient presented with a non- $\mathrm{Q}$ wave acute myocardial infarction (patient with preoperative Type-A aortic dissection and occlusion of the right coronary). Two patients developed a temporary disturbance in renal function but recovered completely.

\section{Discussion}

Nowadays the subclavian artery is our preferred site for cannulation when dissection involves the innominate artery, thus avoiding the complications of a second surgical incision. Dissection is still the bigger issue, but the subclavian artery usually is not involved in the dissection process, although is generally more fragile than the femoral artery and cannulation 
can therefore be traumatic. ${ }^{6,7}$ In the present series, there were no vascular complications arising from the cannulation of the subclavian artery. Systemic perfusion was in all cases controlled without incident. Closure of the arteriotomy was quick and easy.

\section{Funding}

None.

\section{Conflict of Interest}

The authors declare no conflict of interest related to this article.

\section{Acknowledgment}

None.

\section{References}

1 Rylski B, Czerny M, Beyersdorf F, et al. Is right axillary artery cannulation safe in type A aortic dissection with involvement of the innominate artery? J Thorac Cardiovasc Surg 2016;152(03): 801-807.e1

2 Urbanski PP, Sabik JF, Bachetc JE. Cannulation of an arch artery for hostile aorta. Eur J Cardiothorac Surg 2017;51(01):2-9

3 Kazui T, Washiyama N, Muhammad BAH, Terada H, Yamashita K, Takinami M. Improved results of atherosclerotic arch aneurysm operations with a refined technique. J Thorac Cardiovasc Surg 2001;121(03):491-499

4 Mazzola A, Gregorini R, Villani C, Di Eusanio M. Antegrade cerebral perfusion by axillary artery and left carotid artery inflow at moderate hypothermia. Eur J Cardiothorac Surg 2002;21(05): 930-931

5 Sabik JF, Nemeh H, Lytle BW, et al. Cannulation of the axillary artery with a side graft reduces morbidity. Ann Thorac Surg 2004; 77(04):1315-1320

6 Di Eusanio M, Quarti A, Pierri MD, Di Eusanio G. Cannulation of the brachiocephalic trunk during surgery of the thoracic aorta: a simplified technique for antegrade cerebral perfusion. Eur J Cardiothorac Surg 2004;26(04):831-833

7 Schachner T, Nagiller J, Zimmer A, Laufer G, Bonatti J. Technical problems and complications of axillary artery cannulation. Eur J Cardiothorac Surg 2005;27(04):634-637 